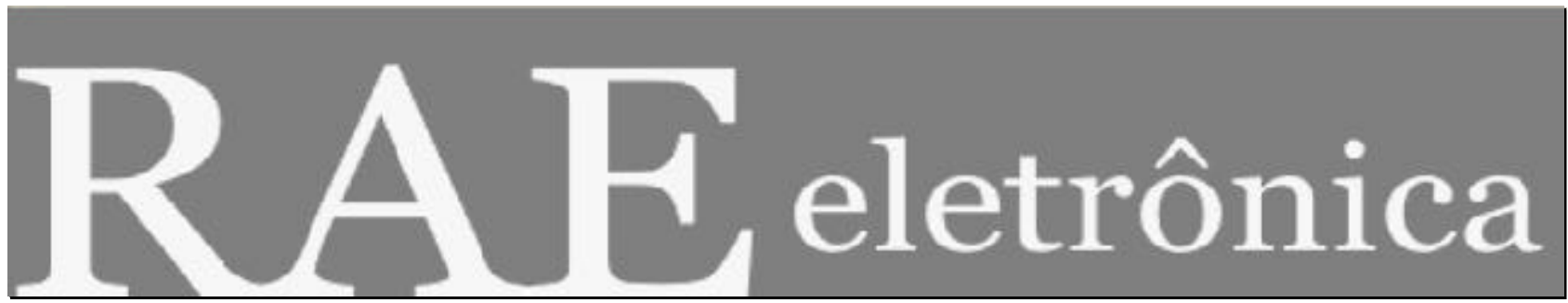

\title{
PLURALIDADE E PENSAMENTO SISTÊMICO EM PROJETOS DE TELECOMUNICAÇÕES
}

Por:

Giovanni Moura de Holanda, $\mathrm{CPqD}$

Esther Menezes, CPqD

Ricardo Benetton Martins, CPqD

Cláudio de Almeida Loural, CPqD

RAE-eletrônica, v. 4, n. 2, Art. 21, jul./dez. 2005

http://www.rae.com.br/eletronica/index.cfm?FuseAction=Artigo \&ID=2249\&Secao=ARTIGOS\&Volu $\mathrm{me}=4 \&$ Numero $=2 \& \mathrm{Ano}=2005$

CCopyright, 2005, RAE-eletrônica. Todos os direitos, inclusive de tradução, são reservados. É permitido citar parte de artigos sem autorização prévia desde que seja identificada a fonte. A reprodução total de artigos é proibida. Os artigos só devem ser usados para uso pessoal e nãocomercial. Em caso de dúvidas, consulte a redação: raeredacao@fgvsp.br.

A RAE-eletrônica é a revista on-line da FGV-EAESP, totalmente aberta e criada com o objetivo de agilizar a veiculação de trabalhos inéditos. Lançada em janeiro de 2002, com perfil acadêmico, é dedicada a professores, pesquisadores e estudantes. Para mais informações consulte o site www.rae.com.br/eletronica.

\section{RAE-eletrônica}

ISSN 1676-5648

(C2005 Fundação Getulio Vargas - Escola de Administração

de Empresas de São Paulo.

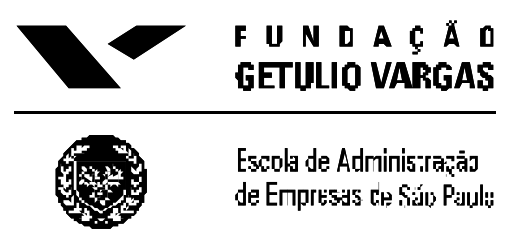




\section{RESUMO}

À medida que aumentam as necessidades de comunicação da sociedade e as relações entre os agentes do mercado se tornam cada vez mais complexas, o planejamento de novos bens ou serviços de telecomunicações requer a adoção de paradigmas sintonizados com o cenário que se delineia. A orientação que tradicionalmente baliza a concepção de novos projetos nesse setor expande-se de um foco técnico-econômico para um panorama que inclua as dimensões sociocultural e político-reguladora. O presente artigo discorre sobre o papel que um paradigma holístico, caracterizado por uma abordagem sistêmica e por uma visão pluralista, desempenha na concepção e na análise de viabilidade de projetos inovadores em telecomunicações, integrando as vertentes sociotécnica e técnico-econômica de análise. A reflexão aqui conduzida busca destacar a importância que o pensamento sistêmico, conjugado com abordagens de cunho qualitativo e que adotam múltiplas perspectivas, exerce em um contexto de análise ex ante de projetos de natureza complexa.

\section{ABSTRACT}

As societal needs for communications increase and relations between market agents become more and more complex, planning of new products and services in the telecommunications sector requires the adoption of paradigms tuned to the new scenario. The orientation that traditionally framed project conception in this sector now expands from a techno-economical focus to one that includes the sociocultural and political-regulatory dimensions. The present article discusses the role that a holistic paradigm, characterized by a systemic approach and a pluralist vision, plays in the conception and viability analysis of innovative telecommunication projects, comprising both the socio-technical and the techno-economical views. The reflection undertaken here aims to point out the importance that systems thinking, allied to multiple perspectives qualitative approaches, exerts in a context of ex ante analysis of projects of this nature.

\section{PALAVRAS-CHAVE}

Pensamento sistêmico, planejamento, projetos de telecomunicações.

\section{KEYWORDS}

Systemic thinking, planning, telecommunication projects. 


\section{INTRODUÇÃO}

Abordagens tradicionais de concepção, avaliação e seleção de projetos ou, dito de outra forma, metodologias de planejamento que consideram apenas os aspectos técnico-econômicos que cercam o objeto de estudo têm se revelado ineficazes no trato das mudanças que acompanham a evolução da sociedade. Tais deficiências ficam mais evidentes à medida que o setor de serviços ganha maior peso na economia, marcado, sobretudo, com a expansão paulatina do nível de informatização da sociedade e com a própria maturidade das relações de mercado.

No setor de telecomunicações, os projetos que pautam o planejamento estratégico das empresas são formatados como bens ou serviços que, para se concretizarem, demanda m recursos financeiros não mais tão abundantes quanto ao tempo da chamada "bolha" de telecomunicações e Internet. Como conseqüência, o planejamento estratégico deve buscar resultados que atendam a expectativas mais rigorosas de retorno de investimento.

Pela própria natureza do setor de serviços, especialmente nas áreas que incorporam tecnologias de informação e comunicação (TICs), o envolvimento dos usuários é fundamental no desenho dos novos projetos, bem como no êxito da sua implantação. Além disso, uma vez introduzidos no ambiente para o qual foram concebidos, os bens ou serviços causam impactos sobre dimensões que transcendem a esfera individual, como, por exemplo, a dimensão socioeconômica e a político-reguladora. Antever a amplitude e os desdobramentos desses impactos não é atividade trivial.

A dificuldade de se obter uma antevisão que efetivamente possa auxiliar o planejamento estratégico é ampliada pelo grau de inovação que o projeto traz em seu bojo, o qual pode estar posicionado ao longo de um espectro que vai do incremental à completa ruptura. Quanto maior o caráter inovador de um projeto, maiores são as incertezas sobre os efeitos de sua introdução no mercado, o que torna a analogia com dados históricos um recurso pouco efetivo para aumentar a previsibilidade e mitigar a opacidade que se apresenta ao analista.

Na difusão de inovações, atuam fatores que são dificilmente quantificáveis ex ante ${ }^{1}$, em função da complexidade e das incertezas circunscritas ao processo de concepção e implantação de um novo projeto. Conseqüentemente, a impossibilidade de se prever a extensão e profundidade dos impactos, sobretudo os de natureza econômica e sociocultural, estimula a inclusão de métodos de caráter subjetivo nas análises preditivas, bem como a utilização de uma gama variada de abordagens.

Ademais, avaliar um problema com base na natureza de seus elementos constitutivos não tem sido um expediente efetivo para revelar as propriedades da unidade global. Como destaca Jackson em seu tratado sobre o pensamento sistêmico aplicado à gestão (2000), a relação entre as partes que compõem um sistema pode ser mais importante que cada uma isoladamente, posto que as propriedades emergentes, reveladoras da unidade resultante, surgem da relação entre elas. Morin (2002), por exemp lo, concebe um sistema como unidade global organizada de inter-relações entre elementos, ações e indivíduos.

Sob essa perspectiva, os projetos de telecomunicações e o ambiente que os cercam podem ser caracterizados como sistemas cuja organização é descrita a partir da relação entre elementos, ações e indivíduos, ou, em uma expressão vocabular cara a Latour (1994), entre componentes humanos e não- 
humanos. A presença do humano no sistema, com suas subjetividades e atitudes comportamentais, permite, a fortiori, compreendê- lo como um sistema social.

Quando o foco recai sobre projetos cuja essência é um processo comunicativo (envolvendo emissores e receptores heterogêneos que se alternam nesses papéis), fica ainda mais evidente a intersecção dos sistemas de telecomunicações com os processos relacionais que formam a base do social. Parte significativa dos problemas enfrentados hoje por organizações e sociedade - que abarcam os setores de telecomunicações e das TICs - apresenta natureza complexa e dinâmica, marcada por processos relacionais, conflitos de interesse e acomodação de forças oblíquas. Abordá-los a partir de paradigmas e métodos reducionistas não tem sido um expediente apropriado para desvendar as origens e extensões dos problemas ${ }^{2}$.

Por conseguinte, a análise de viabilidade de projetos dessa natureza requer antes de tudo uma mudança de paradigma, ou de paradigmas, no sentido de prover um tratamento analítico compatível com todas as dimensões que caracterizam o objeto de estudo. A adoção de uma visão integrada de múltiplas perspectivas, para usar uma terminologia adotada por Linstone (1999), constitui um incremento do processo das estratégias de gestão, ajustável ao contexto que dita as agendas das organizações envolvidas com inovações abrangentes e multifacetadas. Além disso, é igualmente importante evitar o isolamento entre os campos do saber, o que Latour (2000) designa como a babel das disciplinas, ou seja, os economistas da inovação ignorando os sociólogos da tecnologia, que, por sua vez, não dialogam co m os engenheiros e assim por diante.

Sob esses aspectos, o pensamento sistêmico combinado a uma pluralidade de abordagens amplia os horizontes do planejamento de projetos e auxilia a gestão estratégica organizacional na exploração de sistemas e contextos complexos - como os que caracterizam o cenário atual de telecomunicações -, repletos de elementos cuja dinâmica inter-relacional normalmente escapa a uma interpretação linear de causa e efeito. Em outras palavras, tal orientação constitui uma alternativa paradigmática que congrega (i) a visão integrada e transdisciplinar do objeto que se pretende analisar, juntamente com (ii) a adoção de abordagens variadas - tanto de natureza qualitativa como quantitativa - típicas do estágio atual do pensamento sistêmico, (iii) a análise das múltiplas dimensões associadas ao problema e (iv) a consideração das perspectivas de todos os agentes envolvidos.

O presente artigo discorre sobre o papel que esse paradigma holístico desempenha no planejamento de bens e serviços inovadores em telecomunicações, apresentando uma metodologia que, com visão pluralista, integra as vertentes sociotécnica e técnico-econômica de análise. A aplicação ao ambiente das telecomunicações, em especial, deve-se à experiência laboral dos autores. Observa-se, todavia, que o paradigma holístico é aplicável a outros setores socioeconômicos igualmente complexos.

A reflexão aqui conduzida busca destacar a importância que o pensamento sistêmico, conjugado a abordagens de cunho qualitativo e que adotam múltiplas perspectivas, exerce em um contexto de análise ex ante de objetos dessa natureza. Enfim, espera-se que essa reflexão contribua no sentido de formatar soluções mais próximas das reais necessidades de todos os agentes que integram a ecologia das telecomunicações - a jusante e a montante do fluxo de agregação de valor. 


\section{A QUESTÃo DA INOVAÇÃo EM SERVIÇOS NO CENÁRIO ATUAL DAS TELECOMUNICAÇÕES}

A abordagem usual da análise de projetos de telecomunicações costuma ter um viés predominantemente de engenharia e de análise econômica. A agenda específica recente contempla, inclusive, a pesquisa de novas técnicas para suportar esta análise, como, por exemplo, o recurso à teoria das opções reais e outras teorias importadas do setor financeiro (Alleman e Noam, 2002).

Essa abordagem encontra apoio em uma percepção da "rede" como uma estrutura essencialmente física, formada por cabos e equipamentos, que suporta a comunicação entre os usuários dos serviços. Tal percepção foi largamente dominante entre as empresas provedoras dos serviços de telecomunicações e entre seu próprio pessoal. Embora esses atores fossem cientes dos papéis econômico e social das telecomunicações, eles não viam esses outros papéis como tão fundamentais quanto a rede física e os serviços proporcionados por ela. Os usuários certamente possuem outra percepção da rede de telecomunicações, uma percepção muito mais calcada em estabelecer ou excluir determinados grupos sociais, porém essa percepção não era incorporada na visão estratégica dos provedores dos serviços de comunicação (Macdonald, 1998).

Esse quadro tem sido profundamente alterado por conta das fortes transformações pelas quais o setor tem passado, tanto de origem tecnológica quanto de origem institucional, com a introdução da competição e da privatização dos serviços. O processo de mudança tecnológica pode ser sintetizado pela palavra "digitalização" - a representação digital dos sinais associados à informação transmitida. A representação digital carrega características de universalidade quanto ao meio de comunicação (media) e de transparência quanto ao processamento, à transmissão e ao armazenamento das informações. Esse fenômeno permitiu a composição de duas áreas distintas: a área tradicional das telecomunicações com a área da informática, disciplina mais recente.

Tal simbiose trouxe conseqüências marcantes para o comportamento individual e coletivo; para a economia e as práticas organizacionais; para o entretenimento e, não com menos importância, para a criatividade. Alguns de seus efeitos já tinham sido corretamente antecipados desde a década de 1970, no relatório francês "A Informatização da Sociedade" (Nora e Minc, 1978). Porém, com a maturação da tecnologia e com os incentivos oferecidos pelos novos quadros reguladores marcadamente liberalizantes, essas transformações foram aceleradas e ensejaram uma rápida criação de novos bens, serviços e negócios suportados por TICs, os quais constituem o presente cenário do setor de telecomunicações ${ }^{3}$.

Esse processo tem evidenciado uma mudança de percepção fundamental quanto à natureza da inovação nessa área, historicamente marcada por um forte impulso da tecnologia (technology push). Antes de tudo, o setor de telecomunicações é essencialmente um setor de serviços, o que necessita de um novo ferramental de gestão. Com efeito, a tecnologia deve ser encarada como um dos componentes da estrutura de fornecimento de um serviço, sendo apenas parte do problema de introdução de novas ofertas aos usuários. Como já observava Mitchell (1989) há mais de uma década, o setor de serviços é, de fato, um grande cliente da indústria manufatureira, mas esta provê tão somente os elementos da infra-estrutura dos serviços. Esses elementos precisam ser organizados e integrados em um sistema que sirva de suporte ao serviço, o qual é executado ou operado sobre essa infra-estrutura. Além disso, como o cliente direto de um serviço é, na maioria das vezes, o público em geral ou mesmo um indivíduo, a 
interação do usuário com o serviço deve ser parte essencial dessa operação. Revela-se aí o triângulo que caracteriza um serviço (em particular, de telecomunicações), o qual já dá uma medida da complexidade do processo de inovação a ele associado: a infra-estrutura física de suporte; os procedimentos e processos organizacionais; e a relação interativa entre o provedor e o usuário do serviço.

Leijten (1997, 2001) avançou mais na análise do problema da inovação em serviços de telecomunicações. Segundo ele, o desenvolvimento e a introdução de um novo serviço no contexto da sociedade da informação é um processo que envolve uma intensa interação entre o desenvolvimento da tecnologia, as estruturas organizacionais que precisam ser modificadas para o provimento do serviço e, finalmente, o mercado ou seus usuários diretos, implicando, por exemplo, na participação dos usuários potenciais em experimentos e testes durante o desenvolvimento dos novos bens e serviços. Essa interação é adicionalmente complicada pela freqüente necessidade de cooperação entre várias empresas e entidades, bem como pelas disposições legais e reguladoras do Governo, constituindo, portanto, um processo de conjugação de culturas e interesses muitas vezes bastante distintos. Novas atividades devem ser introduzidas nos projetos, como, por exemplo, experimentos reais com uma amostragem dos futuros (ou potencialmente futuros) usuários dos serviços.

Nesse contexto, a necessidade de novas abordagens metodológicas ganha ainda mais relevo quando se trata de conceber e avaliar projetos inovadores, para os quais é fundamental traduzir o conteúdo tecnológico emergente em valor de interesse efetivo para os indivíduos que irão usufruir dessa inovação, resultando, em última análise, na absorção ou incorporação dessa tecnologia como parte do acervo econômico e cultural daquele grupo social dos usuários.

Essa nova visão das telecomunicações, que traz os usuários dos serviços para dentro do próprio planejamento, tornando-os propriamente sujeitos do processo mais do que meros elementos passivos, não é apenas o resultado de constatações empíricas. Ela deita raízes no pensamento das diferentes correntes que estudam a questão da "formatação social da tecnologia" (social shaping of technology), expressão da disciplina sociotécnica. Tais correntes têm mostrado que a tecnologia não evolui apenas segundo uma ló gica interna à técnica, mas é também um produto das condições e padrões de criação e de uso, logo, um produto social (Williams e Edge, 1996).

As transformações e novas perspectivas descritas nesta seção conduzem à mudança na percepção do significado do termo rede, como se discutia anteriormente. Há aqui uma ampliação conceitual do termo, como costumava ser usado, de modo a abranger agora o domínio das relações sociais entre os atores-usuários dos serviços de telecomunicações, sejam eles pessoas ou organizações. Em outras palavras, pelo menos em parte - já que essa percepção ainda não está amplamente difundida -, compreende-se agora a relevância dos papéis não-técnicos da rede, dos papéis de natureza econômica e social. O êxito de uma nova tecnologia e dos serviços de telecomunicação que ela proporciona depende fundamentalmente da satisfação dos seus usuários, satisfação esta que expressa as interações e transações que a nova tecnologia lhes permite estabelecer no plano social. Essas interações entre os inúmeros agentes envolvidos no provimento e na fruição dos serviços de telecomunicações constituem uma das principais características que levam à necessidade de analisar as telecomunicações sob a óptica do pensamento sistêmico e da teoria da complexidade. 


\section{ABORDAGEM SISTÊMICA}

Dado que todos os problemas, especialmente os de natureza plural e complexa, são constituídos de partes interconectadas e, muitas vezes, indissociáveis, pode-se esperar que a relação entre elas e a observação do comportamento global resultante revelem mais informações do que a análise das partes em si. De uma maneira geral, os procedimentos e recursos providos pelo experimentalismo - os quais são majoritariamente conduzidos em laboratórios - prestam-se a testar as relações de causa e efeito entre algumas partes do sistema, o que não chega a ser representativo em termos de revelar explicações para o comportamento de problemas do universo do cotidiano. Mesmo com o aporte dos recursos matemáticos empregados, por exemplo, na teoria de controle, esses métodos mostram-se pouco eficientes para representar as relações entre um número muito grande de elementos e, normalmente, desconsideram a não-linearidade e os efeitos de retroalimentação entre eles. Além disso, muitas condições que cercam os problemas dessa natureza - principalmente dos sistemas sociais - são de difícil reprodução em laboratório, quando não impossíve $4^{4}$.

A dificuldade que os métodos de análise segmentada impõem ao estudo dos problemas complexos é ampliada quando o foco recai nos componentes sociais e, sobretudo, no comportamento humano. Como bem aponta Morin, o conhecimento não é insular, mas peninsular, portanto, para alcançá-lo é necessário ligá-lo ao continente de que faz parte e não o dissociar da condição humana e da relação social (1999, p. 26). De fato, os sistemas sociais apresentam um elevado grau de complexidade e aspectos de difícil mensurabilidade.

A existência do comportamento humano em um sistema, refletida, por exemplo, no exercício da livre vontade, traz dificuldades a processo de modelagem e, uma vez desconsideradas na análise, podem conduzir a resultados não-condizentes com a realidade. Pode-se notar que existem forças internas (preferências, valores, percepções) e externalidades (conjuntura macroeconômica) influenciando e moldando o comportamento dos sistemas que incluem os seres humanos. Nesse campo em particular, a antropologia de consumo e a economia comportamental têm ampliado seu espaço de aplicação, participando de metodologias inusitadas na área de marketing e na própria psicologia ${ }^{6}$.

É se contrapondo às limitações dos métodos reducionistas que surgiram as abordagens sistêmicas que buscam uma visão integrada do problema em estudo. Muitos autores definem essa visão como holística, pela atenção empregada ao todo e às propriedades que resultam da relação entre as partes, muitas vezes, contra-intuitivas (Forrester, 1971).

De um ponto de vista histórico, as teorias que deram sustentação à abordagem sistêmica remontam à cibernética e à teoria geral de sistemas (Jackson, 2000). Vários ramos do conhecimento foram influenciados por essas teorias, não só no campo das ciências naturais como também das ciências humanas. Impulsionaram desde novas práticas de gestão organizacional e disciplinas de engenharia até o trabalho dos estruturalistas. A partir da metade do século passado, a abordagem sistêmica veio a ser largamente adotada na teoria da organização e gestão. Por ser holística, ao considerar as organizações como sistemas inteiros, compostos de partes inter-relacionadas, essa abordagem permitiu cobrir as lacunas dos métodos tradicionais - reducionistas e focalizados em tarefas e estruturas. Um ponto importante na diferença com as abordagens vigentes estava no fato de que a sistêmica passava a considerar as organizações como sistemas abertos, portanto, sob a influência do meio externo, o que é naturalmente peculiar aos problemas do mundo real. 
Entretanto, até a década de 1970, a abordagem sistêmica ainda era dominada pela óptica funcionalista, de forte viés positivista. Só a partir da década de 1980, sobretudo em função da divulgação das teorias do caos e da complexidade, o pensamento sistêmico ganhou novos rumos, constituindo duas vertentes designadas por "pensamento sistêmico soft", no início daquela década, e "pensamento sistêmico crítico", no fim dos anos de 1980 e início dos de 1990 (Jackson, 2000).

De acordo com esse autor, entre os paradigmas da teoria social que exerceram influência na abordagem analítica dos sistemas, o interpretativo constituiu a base teórica para a tendência soft, a qual se apresentou como alternativa à abordagem funcionalista da primeira fase do pensamento sistêmico. No paradigma soft, a ênfase se desloca mais para a dimensão humana do que para os aspectos tecnológicos e estruturais, considerando as múltiplas percepções e valores dos indivíduos. Essa linha incorpora uma visão pluralista de análise, sendo instrumentalizada em abordagens, como, por exemplo, a Quinta Disciplina de Senge e a dinâmica de sistemas de vertente soft, comentadas mais adiante.

O pensamento sistêmico crítico, por sua vez, reforça essa tendência e incorpora procedimentos de debate e diálogo, objetivando o reconhecimento intersubjetivo, na forma de um acordo racional entre os atores envolvidos. Essa orientação dialogal e "terapêutica", juntamente com o uso pluralístico de abordagens e metodologias, está na essência da tendência "crítica" que imprime novos rumos ao pensamento sistêmico.

Apesar de essas tendências terem suscitado certa disputa por uma hegemonia teórica do próprio pensamento sistêmico, elas são consideradas pelos autores deste artigo como sendo complementares e evolutivas, contribuindo para um processo cumulativo de refinamento analítico. Como conseqüência, novos paradigmas e desenvolvimentos teóricos têm dado o atual recorte ao pensamento sistêmico, entre eles:

- A dinâmica de sistemas, iniciada nas décadas de 1950 e 1960 por Jay Forrester (Sterman, 2000), ainda muito confinada à teoria de controle e que no início da década de 1990 ganhou novo apelo na área de gestão com a abordagem de aprendizado organizacional tratada na Quinta Disciplina de Senge (2000), e com a dinâmica de sistemas soft, incluindo, por exemplo, a construção de modelos por grupos multidisciplinares, em vez de um exercício conduzido por um único especialista na técnica (Venix, 1996);

- A abordagem sociotécnica, empenhada, entre outras coisas, em avaliar os impactos que uma inovação pode trazer aos vários setores da sociedade: indivíduos, organizações, indústrias, órgãos reguladores;

- Novas técnicas de construção de cenários e de estudos sobre o futuro tecnológico, desenvolvidas em função da complexidade dos eventos e de suas relações de causa e efeito, quase sempre nãolineares, bem como da incerteza que cerca a ocorrência dos fenômenos, dificultando a previsibilidade do futuro. Desse modo, a abordagem para a construção de cenários foi se modificando para dar espaço ao emprego de novos conceitos e abordagens, incorporando métodos qualitativos e discursivos, e considerando que a inovação tecnológica não é uma ocorrência isolada (Castells, 1999), dependendo de aspectos socioculturais, mercado, conjuntura econômica;

- A modelagem e simulação de fenômenos emergentes como a $\mathrm{ABMS}^{6}$, com aplicações em biologia, ciências sociais, economia e finanças, física e telecomunicações; 
- E o trabalho transdisciplinar de Kahneman e Smith, que une os universos da economia e psicologia ao buscar entender o que pode condicionar, por exemplo, o impulso de compra nos indivíduos. A questão do comportamento não-previsível no processo de decisão é tratada, por exemplo, em Kahneman (2001).

Todos esses aspectos são ainda mais relevantes para as inovações no setor de serviços, especialmente os que envolvem uso intenso de TICs. Assim, na abordagem sistêmica aqui defendida, referente ao planejamento e à análise de viabilidade de projetos de telecomunicações, emprega-se: uma visão integrada das várias dimensões que envolvem o problema, isto é, a sociocultural e mercadológica, a político-reguladora, a econômico-financeira e a técnico-operacional; a inclusão das perspectivas de todos os agentes envolvidos na cadeia de valor do projeto em questão; a transdisciplinaridade em termos de conhecimento para o emprego dos métodos que permitem a condução das análises, e a estruturação coerente e dinâmica das metodologias, métodos, técnicas e modelos que podem ser combinados para planejamento e análise de um projeto particular.

\section{ABORDAGEM PLURALISTA}

De uma maneira geral, entende-se que, para integrar diferentes aspectos de um mesmo problema como o da viabilidade de um projeto de telecomunicações ou de TICs -, o caminho mais adequado passa pelo emprego de uma combinação de técnicas quantitativas e qualitativas, visando elaborar os cenários que acomodarão os bens ou serviços, bem como avaliar a extensão dos impactos que essa nova situação trará para os vários tipos de agentes envolvidos.

Tal situação requer, portanto, uma metodologia de análise que permita tratar os projetos de telecomunicações como sistemas sociais, dinâmicos e abertos, ou seja, relacionando-se com o ambiente que o abarca. Entender tais sistemas, contextualizando seus objetos constituintes, requer um tratamento compatível com a complexidade dos fenômenos que emergem.

Para tanto, é essencial que essa metodologia seja capaz de conjugar não apenas os aspectos técnicos e econômico-financeiros na análise, mas também os de caráter sociocultural envolvidos na introdução de uma nova tecnologia na sociedade. Além disso, devem proporcionar uma análise integrada, qualitativa e quantitativa dos diferentes aspectos do problema, bem como detectar sinergias entre eles.

Nesse contexto, os autores adotam abordagens complementares de tratamento, considerando a visão de cada tipo de agente envolvido no projeto, isto é, a análise é conduzida sob múltiplas perspectivas. O que se busca é, ao final da avaliação, proporcionar uma clarificação do problema em questão e suportar as decisões estratégicas.

A necessidade de uma abordagem plural é defendida inclusive por autores com visão heterodoxa sobre o emprego dos métodos. Em seu propalado trabalho sobre o método, em que defende uma forma "anarquista" de construção de conhecimento, Feyerabend (1993, p. 21) ${ }^{7}$ comenta que "um 
cientista que deseja maximizar o conteúdo empírico dos seus pontos de vista e que quer entendê-los claramente deve, para tanto, introduzir outros pontos de vista, ou seja, adotar uma 'metodologia pluralista"”.

\section{MÚLTIPLAS PERSPECTIVAS EM CENÁRIO SISTÊMICO}

Segundo a concepção de Linstone (1999), a caracterização de um problema é feita com base em três perspectivas: a técnica, a organizacional e a pessoal. A primeira corresponde ao ponto de vista daqueles que propõem soluções lastreadas por modelos matemáticos e computacionais, fortemente calcados em informações quantitativas. A segunda, a organizacional, diz respeito aos interesses das instituições, agências reguladoras, grupos e movimentos sociais presentes na sociedade. Por fim, a perspectiva pessoal trata dos interesses e necessidades individuais no processo de tomada de decisão.

Cada perspectiva representa, portanto, um determinado conjunto de atores da sociedade e seus interesses respectivos. De acordo com o autor, é fundamental que as três perspectivas sejam integradas em análises de processo de decisão, isto é, faz-se necessário o emprego de múltiplas perspectivas na solução de problemas que envolvam uma diversidade de atores. Dessa forma, é possível prover, de forma mais adequada, elementos de apoio à decisão e a ações.

De acordo com Linstone, a perspectiva técnica tende a predominar nas primeiras fases do planejamento, cedendo o espaço para as perspectivas organizacional e pessoal nas fases posteriores de ação (1999, p. 59). Normalmente, as questões técnicas são tratadas por modelos e relações causais, prestando-se a um diagnóstico da situação e fornecendo um mapeamento objetivo das possibilidades. No entanto, os métodos de natureza qualitativa têm por função estruturar as idéias em torno dos objetivos dos tomadores de decisão, que, por sua vez, têm origem nos interesses representados pelas perspectivas organizacional e pessoal. São essas duas perspectivas que definem, de fato, o desfecho final.

No processo de agregação de valor dos projetos de telecomunicações e de TICs, encontram-se, de um lado, os usuários e a sociedade. Os primeiros, como consumidores e condicionantes do valor que está sendo gerado; e a sociedade, como parte interessada de um ecossistema que acomoda uma inovação. De outro lado, à montante da cadeia de valor, estão os provedores de serviço; provedores de conteúdo; operadores de rede; fabricantes de terminais de fruição (por exemplo, aparelhos de TV, computadores e terminais telefônicos); fabricantes de equipamentos, de software e de plataformas; anunciantes; provedores de acesso (por exemplo, acesso à Internet e acesso em banda larga); agentes de comércio eletrônico (por exemplo, lojas virtuais) etc. Portanto, é fundamental que as análises de viabilidade considerem tanto o desempenho econômico, as ameaças e as oportunidades de negócio para os agentes produtores de valor como os impactos sociais, políticos e reguladores, atinentes ao governo e à sociedade. 


\section{A COMPLEMENTARIDADE ENTRE OS TRATAMENTOS QUALITATIVO E QUANTITATIVO}

$\mathrm{Na}$ área de planejamento, as críticas dirigidas tanto a abordagens qualitativas quanto a quantitativas remetem ao conflito entre duas amplas vertentes de estudo relativas à estratégia empresarial. Segundo Godet (1994), tais vertentes são representadas por duas escolas de pensamento: a racionalista e a heurística.

A racionalista teve sua força refletida no amplo empre go das ferramentas quantitativas de análise de portfólio e planejamento estratégico ao longo das décadas de 1960 e 1970. Os criadores desse tipo de ferramenta buscavam regras universalmente aplicáveis à análise estratégica, de modo a garantir o sucesso econômico de uma organização. Isso pressupunha a existência de uma racionalidade no comportamento do sistema em que se inserem os planos, o que levou, por exemplo, à crença na possibilidade de se antever cenários futuros com precisão numérica.

Em termos de abançar esse propósito, a insuficiência dos métodos e técnicas com base no pensamento racionalista contribuiu para reforçar uma outra linha de pensamento estratégico, a heurística, cuja idéia principal é a de que o sucesso de uma empresa depende primordialmente do fator humano, traduzido na estrutura organizacional, no comportamento individual e na intuição para tomada de decisão.

Em seu método prospectivo, Godet argumenta que o emprego de métodos exclusivamente quantitativos é insuficiente para se definir uma estratégia, uma vez que normalmente as análises são comprometidas por dados imprecisos, por extrapolações excessivas e por explicações simplistas do futuro pelo passado. Da mesma forma, a elaboração de uma estratégia empresarial não é favorecida ao se ignorar os aspectos técnico-econômicos e ao desconsiderar informações disponíveis. Em outras palavras: "A visão global da análise prospectiva deve ser baseada no pluralismo e na complementaridade de abordagens" (Godet, 1994, p. 47) ${ }^{8}$.

Nos projetos e cenários de telecomunicações essas considerações são evidentes. Assim, a complementaridade de abordagens qualitativa e quantitativa de análise é necessária para conciliar os interesses de todos os agentes envolvidos na concepção de um novo projeto e avaliar os impactos e tendências futuras.

\section{UM LIAME ENTRE ABORDAGENS}

Na Figura 1 é apresentada uma visão geral de como os métodos podem ser instanciados e concatenados na execução do processo de análise de viabilidade. Pode-se notar que a abordagem de caráter mais subjetivo - representada pelas atividades de construção de cenários e de análise descritiva - é interligada à abordagem de natureza técnico-econômica - como a análise de investimentos baseada em indicadores econômico-financeiros - por meio da análise de riscos. Além disso, os métodos de modelagem e simulação, no caso a dinâmica de sistemas e a $\mathrm{ABMS}^{9}$, constituem outro liame entre as 
abordagens, possibilitando municiar a análise de riscos com elementos provenientes de ambas as vertentes.

\section{Figura 1 - Inter-relacionamento de métodos e abordagens na análise de viabilidade}

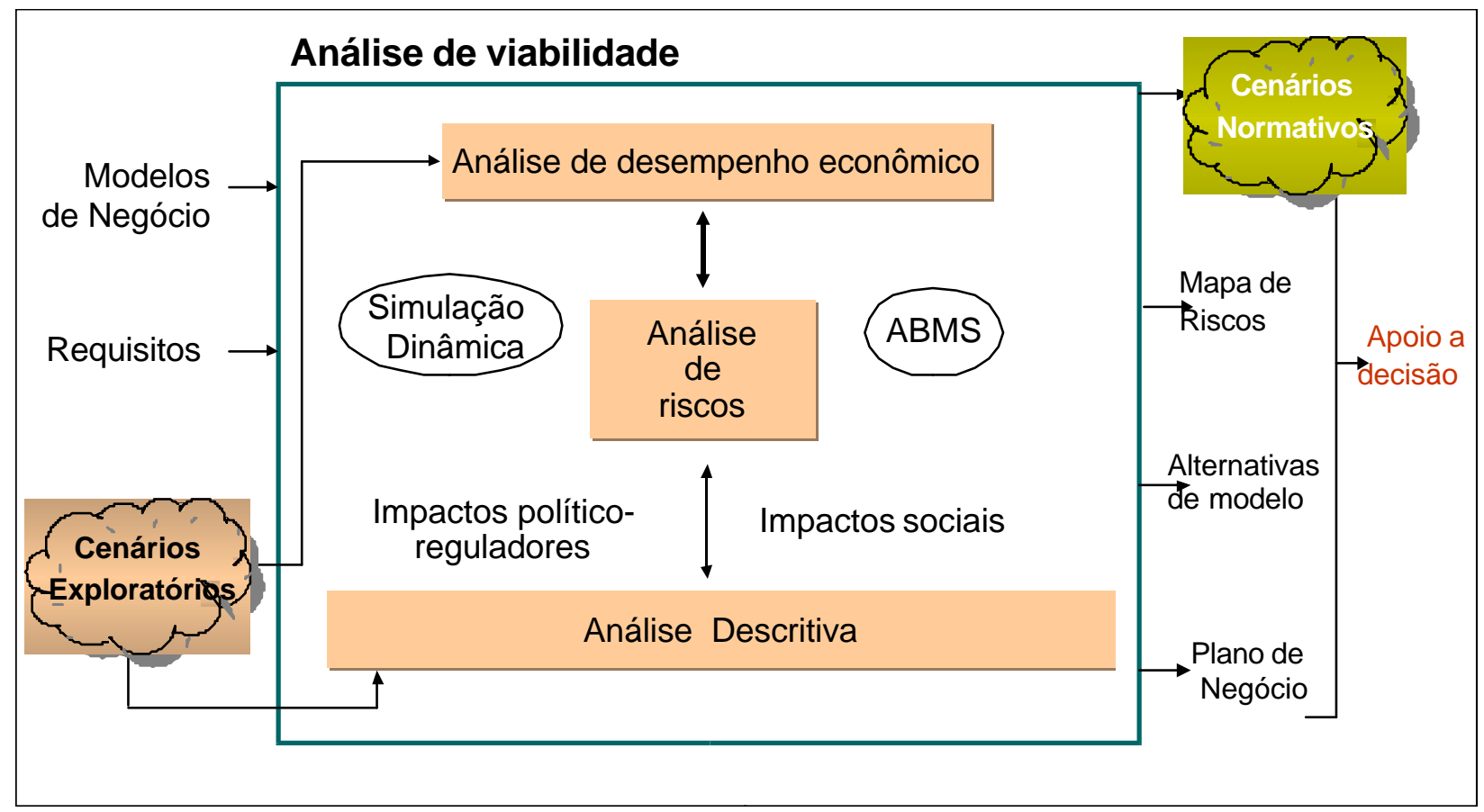

A modelagem e simulação também fornecem meios analíticos para traduzir os cenários exploratórios em cenários almejados ou normativos, conforme a terminologia de cenários empregada por Gordon (1999). Por exemplo, tendo-se por base os cenários macroeconômicos (exploratórios) como pano de fundo, é possível simular o comportamento dos sistemas modelados (cenários normativos) de modo a prover resultados quantitativos sobre os parâmetros de difusão da inovação e o desempenho econômico dos agentes. Por sua vez, os resultados finais desse processo de análise são os insumos para a tomada de decisão quanto à viabilidade do projeto.

No contexto metodológico circunscrito por essa figura, é possível chamar a atenção e trazer à tona alguns detalhes sobre a dinâmica de sistemas e sobre dois métodos de natureza qualitativa, para efeito ilustrativo da metodologia aqui preconizada: o de construção de cenários e o de análise descritiva com base em uma visão sociotécnica do problema em estudo. Os cenários constituem importantes entradas do processo de análise descritiva, o qual se comporta como fio metódico para a condução da análise de riscos e congrega outros métodos e resultados no processo amplo de planejamento e análise estratégica de novos projetos. Entre esses métodos, encontram-se os de natureza técnico-econômica, como aqueles que fornecem estimativas de retorno de investimento: tradicionais - por exemplo, valor presente líquido e taxa interna de retorno - ou sob condições de incerteza e riscos - por exemplo, opções reais. Por restrições de escopo, esses métodos não são aqui tratados. 


\section{Dinâmica de sistemas}

O uso da dinâmica de sistemas no contexto de serviços de telecomunicações vem sendo ensaiado há algum tempo. Pode-se destacar, por exemplo, os trabalhos de Lyons et al. (1995) e de Donaldson et al. (1995) que utilizaram essa abordagem para modelar tanto a competição nos mercados de telecomunicações quanto o ciclo de vida de bens e serviços.

A dinâmica de sistemas consiste na modelagem computacional para simulação do comportamento de sistemas complexos. Um sistema é caracterizado como complexo quando apresenta os seguintes elementos: (i) uma estrutura descrevendo as inter-relações entre as partes, formando o todo; (ii) uma descontinuidade temporal entre decisão, ação e consequiência; e (iii) as amplificações ou retroalimentações, isto é, efeitos cuja intensidade revela-se maior do que o esperado em uma análise simplificada, de primeira ordem (comportamento contra-intuitivo).

Segundo Sterman (2000), o processo organizacional pode ser compreendido como um sistema complexo, posto que se trata de um sistema sujeito a variações em sua dinâmica no decorrer do tempo. Por isso, uma abordagem que incorpora esses conceitos e princípios torna-se adequada ao estudo de atividades de planejamento, uma vez que os modelos mentais normalmente esbarram nas limitações humanas em termos de capacidade cognitiva. Além disso, a aplicação da dinâmica de sistemas viabiliza os testes empíricos de um sistema por meio de simulações, cujos resultados revelam as tendências, tanto as intuitivas como as contra-intuitivas.

\section{Construção de cenários}

A construção de cenários tem sido largamente difundida entre as grandes corporações, em centros de pesquisa e em trabalhos governamentais cujo propósito é o de realizar estudos de prospecção do futuro. Tais estudos, baseados na elaboração de cenários, foram impulsionados a partir da Segunda Guerra Mundial, em função de uma demanda por estudos de monitoração das mudanças sociais por parte de empresas e governos ávidos por entender o futuro do ambiente sociotécnico (Mattelart, 2004). Algumas das técnicas então usadas para construir os cenários do futuro eram: a extrapolação de tendências, os estudos de difusão de tecnologias, a previsão (forecasting) tecnológica e o método Delphi.

Já a análise prospectiva (prospective), termo empregado por Godet (1994) para designar uma abordagem integradora de métodos qualitativos e quantitativos em um único arcabouço metodológico de planejamento estratégico, emprega um método de construção de cenários afinado com o que se convencionou chamar de foresight. A abordagem do tipo foresight representou uma resposta à insuficiência das linhas racionalistas de pensamento - essencialmente deterministas - como abordagens de estudo do futuro tecnológico ${ }^{10}$. Ao mesmo tempo, não ignorou as contribuições dos métodos quantitativos, incorporando-os ao seu arcabouço geral.

Embora possa ser empregada de modo integrado às técnicas quantitativas consagradas, a abordagem foresight de elaboração de cenários é essencialmente qualitativa porquanto fundamentada em duas hipóteses primordiais: (i) o futuro possui uma natureza essencialmente incerta, e (ii) os tomadores de decisão assumem uma postura pró-ativa na formulação e condução dos projetos e de estratégias. A hipótese de indeterminação do futuro implica assumir que há uma ampla diversidade de cenários possíveis de se concretizarem. A hipótese da postura pró-ativa atribui um caráter normativo aos cenários. Conseqüentemente, há espaço para escolhas, que, por sua vez, orientarão as manobras 
para direcionar o curso de um projeto de modo a conduzir à concretização do cenário mais desejável, ou próximo dele, ainda que possa haver restrições ocasionadas por condicionantes dados.

Os cenários obtidos por meio de tal abordagem não ocorrerão de forma irremediável, mas somente sob circunstâncias propiciadas pelas estratégias ditadas pelos próprios tomadores de decisão rumo aos cenários desejados. É uma situação em que as próprias estratégias constituem elementos que compõem os cenários plausíveis interagindo com o ambiente.

Para tanto, a abordagem adotada pelos autores na elaboração de cenários é inspirada nos princípios da análise prospectiva e do foresight, sendo essas duas abordagens integradas à modelagem baseada na dinâmica de sistemas. Essa aplicação integrada se faz em uma seqüência de quatro etapas genéricas:

- Identificação das principais variáveis e dos atores envolvidos. As variáveis são divididas em duas categorias: (i) as exógenas, que compõem os chamados cenários exploratórios e representam o contexto em que o projeto se inserirá; (ii) as endógenas, que compõem os cenários normativos e refletem os elementos passíveis de modificações deliberadas, por parte dos formuladores do projeto em questão. Os cenários normativos são condicionados pelos exploratórios, porém estes, por hipótese, não sofrem realimentação dos resultados das variáveis endógenas.

- Identificação das inter-relações entre essas variáveis e da cadeia de valor na qual se inserem os atores.

- Descrição quantitativa dos cenários exploratórios por meio da integração das variáveis exógenas em um sistema isolado e projeção de suas trajetórias hipotéticas e seus respectivos estados finais ou desfechos. Para essa etapa, pode-se recorrer ao apoio de modelos estatísticos, econométricos ou mesmo de simulação dinâmica.

- Seleção dos cenários exploratórios mais factíveis para que sirvam como pano de fundo ao processo de simulação dinâmica e teste dos cenários normativos.

\section{Análise descritiva}

A análise descritiva tem se caracterizado como um método discursivo apropriado a contextos marcados por incertezas, permitindo um trato mais satisfatório de questões de difícil mensurabilidade. É essencialmente baseada na prática de diálogos com vistas ao aprendizado e à busca de um entendimento comum entre os agentes envolvidos com o sistema em análise. Trata-se, portanto, de uma abordagem alinhada com a vertente do pensamento sistêmico crítico, conforme já mencionado.

Autores como Renn (1999) e Van der Heijden (2000) têm defendido tal tipo de abordagem, e este último aponta também um relacionamento estreito entre os métodos discursivos e a construção de cenários quanto à troca de impressões e de argumentos sobre as possíveis causas geradoras de ação dos indivíduos. Nesse contexto, uma metodologia de planejamento deve incorporar procedimentos sistemáticos de diálogo, buscando avaliar qualitativamente um projeto com base na integração das diferentes perspectivas.

Isso posto, a metodologia de análise de viabilidade aqui descrita incorpora um processo de análise descritiva que, por meio de um diálogo sistemático, busca avaliar adequadamente um projeto 
com base na integração das diferentes perspectivas. Tal processo de análise pode ser empregado ao longo de todo o ciclo de vida de um projeto: desde a sua concepção, passando por todo o processo de planejamento, até a sua implementação e as decisões sobre sua continuidade. A análise descritiva atua como balizador da construção e análise dos cenários, no que diz respeito à definição de seus pressupostos. Permite também refinar os requisitos do projeto, a partir de uma visão sistêmica e pluralista, além de detectar possíveis problemas futuros na sua trajetória. A análise descritiva é baseada em um processo de aprendizagem, sendo conduzida por meio de sucessivas descrições do projeto, a partir de um procedimento socioterapêutico entre o proponente (projetistas) e o avaliador ${ }^{11}$. As diferenças entre as descrições permitem apontar erros e desvios típicos ${ }^{12}$ da fase de planejamento, contribuindo para o enriquecimento do projeto.

\section{UM EPÍTOME DA VISÃO PLURAL E DO TRATAMENTO SISTÊMICO}

As tecnologias de telecomunicações, e sua extensão nas novas tecnologias de informação e comunicação, possuem um caráter intrinsecamente plural, de forte natureza sociotécnica, compreendendo aspectos críticos além daqueles apenas tecnológicos ou econômicos. Essa constatação recomenda o emprego de abordagens não-convencionais na avaliação ex ante de projetos nesses setores, em particular aqueles com elevada dose de inovação, seja na tecnologia em si, seja no seu uso ou aplicação. Busca-se, dessa forma, tratar os projetos e o ambiente que o abarca como sistemas e redes sociais, condicionados e condicionantes de fatores político-reguladores, sociológicos e até mesmo psicológicos.

A discussão aqui apresentada concentrourse em abordagens que adotam o pensamento sistêmico com uma visão sob múltiplas perspectivas do projeto, destacando-se os recursos da dinâmica de sistemas (de vertente soft), das técnicas de construção de cenários e da análise descritiva. Em conjunto com as técnicas tradicionais de análise econômico-financeira e de riscos, elas compõem um leque de métodos e técnicas complementares que vêm sendo empregados, de modo sistemático, pelos autores e seus colegas, em estudos de viabilidade de projetos voltados ao desenvolvimento de novos serviços de telecomunicações ou à introdução de novas tecnologias.

Como ilustração de aplicações dessa abordagem, frutos da experiência dos autores, é possível destacar alguns empreendimentos e trabalhos de consultoria. O planejamento de um produto voltado a serviços e aplicações multimídia e de um serviço de intercâmbio seguro de mensagens assíncronas que se encontram em fase de comercialização e operação. O planejamento de dois serviços baseados em tecnologia digital e interativa por meio de radiodifusão: um para aplicações de educação a distância e outro para acesso em banda larga - ambos se encontram em fase final de análise de viabilidade. Consultorias para órgãos do governo, envolvendo, por exemplo, a análise de viabilidade de um novo serviço para universalização do acesso a redes digitais e a análise de um modelo de referência para implantação da TV digital terrestre no Brasil.

Em cada uma dessas aplicações, foi selecionada e empregada uma pluralidade de métodos e técnicas, compatíveis com a natureza do objeto de estudo e com o escopo da análise - todos, evidentemente, em sintonia com a abordagem sistêmica. No caso particular de ino vações radicais, em que as dificuldades inerentes ao projeto são ampliadas por falta de dados históricos, a análise 
descritiva, por exemplo, proporcionou uma visão mais nítida sobre as perspectivas dos agentes envolvidos e facilitou a identificação de possíveis problemas futuros. Tais benefícios analíticos ficaram evidentes nos casos do serviço voltado para educação a distância e de um conversor texto-fala (outro projeto desenvolvido pela instituição dos autores). A construção de cenários, por sua vez, foi empregada em todos os exemplos citados, tanto em planejamento quanto em consultorias de análise estratégica, permitindo aumentar a previsibilidade dos impactos associados aos projetos e municiar os modelos de simulação e a análise de viabilidade correspondente. ${ }^{13}$

Todavia, é importante mencionar algumas limitações de uma abordagem como essa. Em primeiro lugar, a dificuldade de explicitar os benefícios e de contextualizar os clientes e interlocutores quanto ao novo paradigma de análise. A heterogeneidade de visões a esse respeito, sobretudo nas fases iniciais de análise, exige dos analistas um esforço adicional para proporcionar um ambiente sinérgico, favorável à aprendizagem e à consecução dos objetivos propostos. Deve-se, portanto, considerar esses aspectos no dimensionamento de cronograma e na alocação de recursos para a homogeneização de conceitos e para a condução dos processos de diálogo e debates, quando aplicáveis.

Outra dificuldade está no levantamento de dados de entrada com alto teor de subjetividade, principalmente em se tratando de inovações radicais. Esse aspecto vem a lume quando (i) se tenta caracterizar demanda e sensibilidade a preço para bens e serviços sem equivalentes na percepção dos usuários ou (ii) diante da identificação de possíveis riscos, das chances de ocorrência e da extensão dos impactos a eles associados. Esse não é um problema exclusivo da abordagem sistêmica, mas é acentuado pela adição substancial dos fatores subjetivos à analise. A complementaridade de abordagens de modelagem e simulação tem sido útil em termos de contornar a dificuldade de obtenção de dados, revelando insights de fenômenos emergentes que podem ser ocultados por uma única tratativa de abordagem. Ademais, a inclusão das perspectivas dos múltiplos agentes e a composição de equipes multidisciplinares de análise têm contribuído para aumentar a previsibilidade acerca dos riscos e vicissitudes atrelados a uma inovação.

Em síntese, a pluralidade de versões da abordagem sistêmica, associada a uma multiplicidade de perspectivas (ou de saberes, por que não?), pode ser considerada como um processo de amadurecimento analítico, que, mesmo sem permitir uma apreensão total dos fenômenos, proporciona maiores chances de entendimento das questões do mundo real.

\section{NOTAS}

${ }^{1}$ Embora alguns modelos matemáticos de representação do mercado, como o de Bass (1969), possuam parâmetros quantitativos que incorporam fatores de natureza subjetiva, por exemplo, o que representa o grau de atratividade de uma inovação.

2 Uma discussão dos problemas decorrentes dessa separação é apresentada em Latour (1994), ressaltando a questão de que os sistemas são constituídos de componentes humanos e não-humanos e 
que precisam ser tratados de forma conjunta; e em Sterman (2000), no contexto do pensamento sistêmico e da dinâmica de sistemas aplicados aos negócios.

${ }^{3}$ Uma interpretação dessas mudanças na estrutura econômica e organizacional do setor pode ser encontrada em Fransman (2001).

${ }^{4}$ Além dessas, Jackson (2000) e Sterman (2000) levantam, com um nível razoável de detalhes, uma série de deficiências atreladas aos métodos reducionistas de investigação científica e de modelagem de sistemas no trato de problemas complexos.

5 No tocante à contribuição da economia comportamental para o entendimento das inovações no ambiente das telecomunicações, ver, por exemplo, Ogushi et al. (2004).

6 Agent-Based Modeling and Simulation (modelagem e simulação baseada em agentes). Essa abordagem, diferentemente da dinâmica de sistemas, que parte do comportamento geral (top-down), é concebida a partir do comportamento dos agentes, cujas inter-relações determinam a emergência dos fenômenos que se deseja observar. Para mais informações sobre esse tipo de modelagem, ver, por exemplo, Tyson (1997) ou Epstein e Axtell (1996).

7 Tradução livre do original em inglês.

${ }^{8}$ Tradução livre do original em inglês.

${ }^{9}$ Uma aplicação da modelagem por agentes, bem como da dinâmica de sistemas, na avaliação do comportamento heterogêneo dos indivíduos em um mercado em que se introduz uma inovação em serviço de telecomunicações é apresentada em Holanda et al. (2003a).

${ }^{10}$ Exemplos de atividades de foresight realizadas em alguns países podem ser encontrados em Coates (1999) (sobre os EUA), e Grupp e Linstone (1999).

${ }^{11}$ Nesse ponto é importante notar a semelhança com os procedimentos de diálogo do pensamento sistêmico crítico, como anteriormente mencionado.

12 Uma descrição desses erros e desvios foi desenvolvida no âmbito do projeto PROTEE - uma metodologia de avaliação de inovações, que contou com a participação do grupo de sociologia da inovação da Ècole des Mines de Paris.

${ }^{13}$ Alguns dos resultados alcançados nessas aplicações são comentados em Holanda et al. (2003b).

Este trabalho foi realizado com o suporte do FUNTTEL - Fundo para o Desenvolvimento Tecnológico das Telecomunicações, no âmbito dos projetos Tecnologias de Serviços de Telecomunicações (encerrado) e Cenários Tecnológicos de Telecomunicações (em andamento). 


\section{REFERÊNCIAS}

ALlEMAN, J.; NOAM, E. The New Investment Theory of Real Options and its Implication for Telecommunications Economics. Boston: Kluwer Academic Publishers, 2002.

BASS, F. M. A new product growth model for consumer durables. Management Science, v. 15, n. 5, jan. 1969.

CASTELLS, M. A Sociedade em Rede. São Paulo: Paz e Terra, 1999.

COATES, V. Technology forecasting and assessment in the United States: statistics and prospects. Futures Research Quarterly, v. 15, n. 3, 1999.

DONALDSON, C. F.; SKELTON, S; LYNCH, T; LYONS, M. H. Modelling product life cycles from customer choice. European Transactions in Telecommunications, v. 6, p. 431-7, 1995.

EPSTEIN, J. M.; AXTELL, R. L. Growing artificial societies: social science from the bottom up. Washington: The Brookings Institution/MIT Press, 1996.

FEYERABEND, P. Against Method. London: Verso, 1993.

FORRESTER, J. Counterintuitive behavior of social systems. Technology Review, jan. 1971.

FRANSMAN, M. Evolution of the telecommunications industry into the internet age. Communications \& Strategies, n. 43, p. 57-113, 2001.

GODET, M. From anticipation to action: a handbook of strategic prospective. Paris: Unesco Publishing, 1994.

GORDON, T. J. Integration of forecasting methods and the frontiers of future research. In: GLENN, J. C. (ed.) Futures Research Methodology. Washington: American Council for The United Nations University - The Millenium Project, 1999.

GRUPP, H.; LINSTONE, H. A. National technology foresight activities around the globe: resurrection and new paradigms. Technological Forecasting and Social Change, v. 60, n. 1, p. 85-94, 1999.

HOLANDA, G. et al. Modelling the Bass Diffusion Process Using An Agent-Based Approach. In: WORKSHOP ON AGENT-BASED SIMULATION, 4., 2003, Montpellier. Proceedings. SCS-Europe, 2003a.

HOLANDA, G. M. et al. Planejamento e avaliação estratégica de projetos de telecomunicações: uma perspectiva metodológica. In: CONGRESSO IBERO-AMERICANO DE GERÊNCIA DE PROJETOS, 4., 2003, São Paulo. (b)

JACKSON, M. C. Systems approaches to management. New York: Kluwer Academic/Plenum, 2000.

KAHNEMAN, D.; SLOVIC, P.; TVERSKY, A. Judgment under uncertainty: heuristics and biases. New York: Cambridge University Press, 2001.

LATOUR, B. Jamais fomos modernos: ensaio de antropologia simétrica. Rio de Janeiro: Editora 34, 1994. 
LATOUR, B. Ciência e ação: como seguir cientistas e engenheiros sociedade afora. São Paulo: UNESP, 2000.

LEIJTEN, J. R\&D for services in information society. Research and development policies, new industrial deal and European challenges. Montpellier: IDATE, 1997.

LEIJTEN, J. Public experimentations for new ICT markets. Communications \& Strategies, n. 44, p. 145-70, 2001.

LINSTONE, H. A. Decision making for technology executives: using multiple perspectives to improve performance. [S.1]: Artech House, 1999.

LYONS, M. H.; LYNCH, T.; SKELTON, S. Modelling competition in telecommunications markets. European Transactions in Telecommunications, v. 6, p. 407-14, 1995.

MACDONALD, S. Notions of network: some implications for telecommunications of differences in perception. In: MACDONALD, S. \& MADDEN, G. (eds). Telecommunications and Socio-Economic Development. Amsterdam: Elsevier, 1998.

MATTELART, A. A Era da informação: gênese de uma denominação descontrolada. In: MARTINS, F. M.; SILVA, J. M. A Genealogia do Virtual: comunicação, cultura e tecnologias do imaginário. Porto Alegre: Sulina, 2004.

MITCHELL, G. Research and development for services. Research Technology Management, v. 32, $\mathrm{n}$. 6, p. 37-44, nov./dec., 1989.

MORIN, E. O Método 3: o conhecimento do conhecimento. Porto Alegre: Sulina, 1999.

MORIN, E. O Método 1: a natureza da natureza. Porto Alegre: Sulina, 2002.

NORA, S.; MINC, A. L'Informatisation de la société. Paris: La Documentation Française, 1978.

OGUSHI, C. M.; GEROLAMO, G. P.; MENEZES, E.; HOLANDA, G. M. A economia comportamental e a adoção de uma inovação tecnológica no cenário de telecomunicações. In: SIMPÓSIO DE GESTÃO DA INOVAÇÃO TECNOLÓGICA, 23, 2004, Curitiba. Anais. São Paulo: PGT/USP, 2004.

RENN, O. Participative technology assessment: meeting the challenges of uncertainty and ambivalence. Futures Research Quarterly, v. 15, n. 3, p. 81-97, 1999.

SENGE, P. A Quinta Disciplina: arte e prática da organização que aprende. São Paulo: Best Seller, 2000 .

STERMAN, J. D. Business dynamics: systems thinking and modeling for a complex world. Boston: McGrawHill High Education, 2000.

TYSON, P. Artifical societies. Technology Review, p. 15-7, apr. 1997.

VAN DER HEIJDEN, K. Scenarios and forecasting: two perspectives. Technological Forecasting and Social Change, v. 65, n. 1, p. 31-6, 2000. 
VENIX, J. A. M. Group model building: facilitating team learning using system dynamics. Chichester: John Wiley \& Sons, 1996.

WILLIAMS, R.; EDGE, D. The social shaping of technology. Research Policy, v. 25, p. 856-99, 1996.

\section{Artigo recebido em 29.12.2003. Aprovado em 09.02.2005.}

\section{Giovanni Moura de Holanda}

Pesquisador Sênior da Fundação CPqD. Mestre em Engenharia Elétrica pela Unicamp.

Interesse de pesquisa nas áreas de metodologias de análise, pensamento sistêmico e gestão da inovação.

E-mail: giovanni@cpqd.com.br

Endereço: Rod. Campinas - Mogi-Mirim (SP 340) km 118,5, Campinas - SP, 13086-902.

\section{Esther Menezes}

Pesquisadora da Fundação CPqD. Bacharel em Ciências Econômicas pela Unicamp.

Interesse de pesquisa nas áreas de planejamento e estratégia, pensamento sistêmico, dinâmica de sistemas e modelagem baseada em agentes.

E-mail: esther@cpqd.com.br

Endereço: Rod. Campinas - Mogi-Mirim (SP 340) km 118,5, Campinas - SP, 13086-902.

\section{Ricardo Benetton Martins}

Diretor de TV Digital da Fundação CPqD. Doutor em Ciência dos Materiais pela Université de Paris XI.

Interesse de pesquisa nas áreas de gestão da inovação, planejamento e estratégia.

E-mail: benetton@cpqd.com.br

Endereço: Rod. Campinas - Mogi-Mirim (SP 340) km 118,5, Campinas - SP, 13086-902.

\section{Cláudio de Almeida Loural}

Gerente de Planejamento da Inovação da Fundação CPqD. Mestre em Ciências dos Materiais pelo Instituto Militar de Engenharia - IME.

Interesse de pesquisa nas áreas de gestão da inovação, planejamento e estratégia.

E-mail: loural@cpqd.com.br

Endereço: Rod. Campinas - Mogi-Mirim (SP 340) km 118,5, Campinas - SP, 13086-902. 